\title{
Lipoproteína (a) em pacientes portadores de doença arterial obstrutiva periférica e/ou diabetes mellitus tipo 2
}

Primeira submissão em 12/12/05 Última submissão em 09/04/08 Aceito para publicação em 13/05/08 Publicado em 20/04/08

\author{
Lipoprotein (a) in patients with peripheral arterial obstructive disease and/or type 2 diabetes mellitus
}

Ana Paula Lucas Mota ${ }^{1}$; Maria das Graças Carvalho²; Luciana Moreira Lima;

Maria Elisabeth Rennó de Castro Santos ${ }^{4}$; Marinez de Oliveira Sousa ${ }^{2}$

unitermos
Lipoproteína (a)
Perfil lipídico
Doença arterial obstrutiva
periférica
Diabetes mellitus tipo 2

Diabetes mellitus tipo 2

\section{resumo}

Introdução: A doença arterial obstrutiva periférica (DAOP) constitui um excelente marcador para a aterosclerose sistêmica. Entre os fatores de risco para essa doença está o diabetes mellitus tipo 2 (DM2). Acredita-se que a lipoproteína (a) [Lp(a)] esteja ligada a risco aumentado de aterosclerose, embora os mecanismos que levem a esse aumento não sejam completamente conhecidos. Níveis elevados de Lp(a) parecem estar associados a risco aumentado de doença arterial coronariana (DAC), assim como DAOP e doença cerebrovascular. Objetivo: Avaliar os níveis plasmáticos de $L p(a)$ e outras variáveis lipídicas em um grupo de pacientes com DAOP e/ou DM2. Material e métodos: Níveis plasmáticos de Lp(a), colesterol total (CT), colesterol da lipoproteína de alta densidade (HDL-C), colesterol da lipoproteína de baixa densidade (LDL-C), triglicérides (TG) e apolipoproteínas A-I e B foram medidos em amostras de sangue de 12 indivíduos comprovadamente não-portadores de DAOP e DM2 (controles), 17 pacientes portadores de DAOP, 18 pacientes com DM2 e 19 pacientes portadores de DAOP e DM2 simultaneamente. Os participantes desse estudo foram selecionados buscando-se homogeneidade e semelhança estatística em relação às variáveis sexo, idade e nível socioeconômico. Resultados: A Lp(a) apresentou tendência a elevação tanto no grupo de pacientes com DAOP quanto naquele com DM2 + DAOP. Foram encontradas diferenças significativas entre os grupos para as dosagens de HDL-c e Apo A-I, inclusive com correlação positiva entre esses parâmetros. A relação CT/HDL-C apresentou diferença estatística significativa entre os grupos. Foram observadas correlações positiva entre $L p(a)$ e LDL-c, e negativa entre o índice tornozelo-braquial (ITB) e a Lp(a). Conclusão: Para as variáveis lipídicas estudadas foram observadas diferenças estatísticas significativas apenas entre os níveis plasmáticos de HDL-c e Apo A-I. Para o parâmetro $L p(a)$ foram observados níveis plasmáticos mais elevados nos grupos DAOP e DM2 + DAOP, os quais também apresentaram uma concomitante e significativa redução de HDL-c.

\footnotetext{
Background: Peripheral arterial obstructive disease (PAOD) constitutes an excellent marker for systemic atherosclerosis and type 2 diabetes mellitus (DM2) is among the greatest risk factors for this disease. It is believed that lipoprotein $(a)[L p(a)]$ is linked to increased risk of atherosclerosis, although the mechanisms responsible for that are not widely known. Elevated levels of $L p(a)$ seem to be associated with a higher risk of coronary artery disease (CAD), as well as PAOD and cerebrovascular disease. Objectives: To assess the plasma levels of $L p(a)$ and other lipid parameters in patients with PAOD and/or DM2. Material and methods: Plasma levels of $L(a)$, total cholesterol (TC), high-density lipoprotein cholesterol (HDL-c), low-density lipoprotein cholesterol (LDL-C), triglycerides (TG) and apolipoproteins $A-I$ and $B$ were measured in blood samples of 12 subjects carrying neither PAOD nor DM2 (control group), 17 patients with PAOD, 18 with DM2 and 19 with both PAOD and DM2. The subjects selected for this study showed homogeneity and no statistical difference for gender, age, and socioeconomic status. Results: The $L p(a)$ showed a tendency to elevation both in groups $P A O D$ only and PAOD + DM2 simultaneously. Significant differences were observed among the groups as to $H D L-c$ and apolipoprotein A-l levels, with positive correlation between these two parameters. TC/HDL-c ratio showed significant difference among the groups. Positive correlation was found between $L P(a)$ and $L D L-C$, and negative one, between the ankle-arm index and $L P(a)$. Conclusion: As to the lipid parameters studied, significant statistical differences were found between HDL-c and apolipoprotein A-I plasma levels only. For $L p(a)$ parameter, higher plasma levels were observed in PAOD and PAOD $+D M 2$, which have also shown concomitant and significant HDL-c reduction.
}

\section{key words}

Lipoprotein (a)

Lipidic profile

Peripheral arterial

obstructive disease

Type 2 diabetes mellitus

1. Mestre em Ciências Farmacêuticas pela Faculdade de Farmácia da Universidade Federal de Minas Cerais (UFMC).

2. Professoras doutoras do Departamento de Análises Clínicas e Toxicológicas da Faculdade de Farmácia da UFMC.

3. Doutora em Ciências Farmacêuticas pela Faculdade de Farmácia da UFMG.

4. Médica angiologista do Hospital Santa Casa de Misericórdia de Belo Horizonte.

Trabalho realizado no Departamento de Análises Clínicas e Toxicológicas da Faculdade de Farmácia da Faculdade de Farmácia da UFMG. 


\section{Introdução}

De acordo com dados da Organização Mundial da Saúde (OMS)(20), as doenças cardiovasculares (DCVs) estão classificadas em cerca de 69 grupos, incluindo a cardiopatia isquêmica, a isquemia cerebral, o infarto agudo do miocárdio (IAM), o acidente vascular cerebral (AVC), a doença arterial obstrutiva periférica (DAOP) e várias outras relacionadas. Atualmente a aterosclerose é apontada como mecanismo comum para o desenvolvimento de todos esses distúrbios anteriormente citados ${ }^{(9)}$.

A DAOP constitui importante marcador para a aterosclerose sistêmica, atingindo cerca de $12 \%$ da população em geral. Entre os maiores fatores de risco para DAOP está o diabetes mellitus tipo 2 (DM2). Estima-se que cerca de $80 \%$ das causas de morte em indivíduos diabéticos sejam por ocorrência de eventos trombóticos e que $75 \%$ deles sejam eventos cardiovasculares. A ocorrência simultânea dessas duas doenças favorece o desenvolvimento de eventos vasculares isquêmicos devido ao caráter sistêmico de ambas. Além disso, pacientes com DAOP e DM2 podem apresentar alterações do perfil lipídico, com tendência a hipercolesterolemia e hipertrigliceridemia ${ }^{(15)}$.

A associação entre dislipidemia e DAOP não está totalmente esclarecida, porém a relação colesterol total (CT)/colesterol da lipoproteína de alta densidade (HDL-C) foi considerada um marcador preciso para risco de desenvolvimento de doença arteria|(16).

A etiologia da complicação vascular diabética é obviamente multifatorial. O DM2 está associado a uma variedade de complicações de pequenos e grandes vasos como retinopatia, nefropatia, neuropatia, IAM, AVC e doenças em vasos periféricos, que são freqüentemente responsáveis pela morte prematura dos pacientes. Eventos macrovasculares são mais freqüentemente relacionados com processos trombóticos graves, como o desenvolvimento de DAOP(21).

A lipoproteína (a) [Lp(a)] foi descrita por Berg em 1963 como sendo uma variante genética da $L D L$, que apresenta a apolipoproteína $\mathrm{A}(\mathrm{Apo} A$ ) ligada à apolipoproteína $\mathrm{B}$ (Apo B) por meio de pontes dissulfeto ${ }^{(6,10)}$.

A Apo A apresenta alta homologia estrutural com o plasminogênio (PMG), entretanto não tem o mesmo sítio catalítico deste, mas alguns estudos sugerem que sua presença poderia inibir competitivamente a ligação do PMG ao seu receptor, reduzindo, assim, a formação de plasmina e a fibrinólise ${ }^{(7)}$. Todas essas alterações lipídicas, bem como a hipofibrinólise, podem contribuir para o aumento do risco de DCVs.
A função da $L p(a)$ tem sido alvo de grandes estudos, pois seus níveis persistentemente altos no plasma parecem estar forte e independentemente associados à aterosclerose. A $L p(a)$ pode ligar-se à matriz extracelular (MEC), depositando sua lipoproteína de baixa densidade (LDL) associada. Esse seria um dos mecanismos para desenvolvimento da aterosclerose ${ }^{(2,14)}$.

O metabolismo da $L p(a)$ não está totalmente esclarecido, contudo certas anormalidades metabólicas parecem influenciar as concentrações circulantes de $L p(a)$. Esses valores podem ser aumentados como parte da resposta inflamatória de fase aguda no DM2, insuficiência renal crônica, câncer, hipotireoidismo e aterosclerose ${ }^{(14)}$.

O aumento potencial dos níveis plasmáticos de Lp(a) é de especial interesse no processo aterosclerótico que leva à $D A O P$, sugerindo que a concentração de $L p(a)$ poderia ser influenciada pela presença de doença vascular extensi$\mathrm{va}^{(14)}$. A Lp(a) está presente em grandes concentrações em artérias com aterosclerose quando em comparação com as artérias normais, o que contribui para a redução da atividade fibrinolítica na placa aterosclerótica ${ }^{(5)}$.

Nesse contexto, o presente trabalho pretende investigar a hipótese de que a ocorrência concomitante de DAOP e DM2 favorece as alterações do metabolismo lipídico, com posterior hiperlipidemia, o que contribui para o agravamento dessas doenças.

\section{Material e métodos}

Os pacientes com DAOP e/ou DM2 foram selecionados durante a rotina de admissão do Hospital Santa Casa de Misericórdia de Belo Horizonte, entre os internados na enfermaria ou em controle ambulatorial no Serviço de Cirurgia Cardiovascular, sendo de ambos os sexos e com idade compreendida entre 45 e 75 anos, enquanto os indivíduos incluídos no grupo controle foram selecionados da comunidade em geral, com idade similar aos pacientes selecionados.

Os participantes deste estudo de coorte transversal foram selecionados buscando-se homogeneidade e semelhança estatística em relação às variáveis sexo, nível socioeconômico e idade (Tabela 1).

A dislipidemia e a história familiar para DAOP apresentaram distribuição semelhante entre os grupos, entretanto nenhum indivíduo do grupo controle manifestou alterações compatíveis com dislipidemia. Para análise dos fatores de risco foi empregado o teste exato de Fisher. As diferenças 
Tabela 1 Caracterização dos grupos quanto aos fatores de risco para desenvolvimento de DAOP

\begin{tabular}{lccccc}
\hline $\begin{array}{l}\text { Fatores de risco } \\
(\%)\end{array}$ & $\begin{array}{c}\text { Controle } \\
(n=12)\end{array}$ & $\begin{array}{c}\text { DAOP } \\
(n=17)\end{array}$ & $\begin{array}{c}\text { DM2 } \\
(n=18)\end{array}$ & $\begin{array}{c}\text { DM2 + DAOP } \\
(n=19)\end{array}$ & Valor de $p$ \\
HAS & $4(33,3 \%)$ & $15(88,2 \%)^{*}$ & $12(66,7 \%)$ & $12(63,2 \%)$ & $<0,001$ \\
Dislipidemia & 0 & $2(11,8 \%)$ & $3(16,7 \%)$ & $1(5,3 \%)$ & $n$ ns \\
Tabagismo & $2(16,7 \%)$ & $15(88,2 \%)^{*}$ & $6(33,3 \%)$ & $11(57,9 \%) *$ & 0,032 \\
HF & $8(66,7 \%)$ & $11(64,7 \%)$ & $12(66,7 \%)$ & $14(73,7 \%)$ & $n$ ns \\
HP & 0 & $7(41,2 \%)^{*}$ & $2(11,1 \%)$ & $5(26,3 \%)$ & 0,023 \\
Idade (médias) & 58,4 & 62,1 & 60,2 & 64,1 & $n$ \\
Sexo (M/F) & $5 / 7$ & $9 / 8$ & $10 / 8$ & $8 / 11$ & $n$ \\
\hline
\end{tabular}

Os grupos assinalados com *apresentaram diferença estatística significativa em relação ao grupo controle, e os não-assinalados não a apresentaram para os parâmetros avaliados. DAOP: doença arterial obstrutiva periférica; n: número de participantes do estudo; DM2: diabetes mellitus tipo 2; HAS: hipertensão arterial sistêmica; HF: história familiar para doença vascular; HP: história prévia para doença vascular; ns: não-significativo.

estatísticas apresentadas encontram-se ilustradas na Tabela 1. Em relação ao uso de medicamentos, foi constatado que apenas $5 \%$ dos pacientes estudados estavam em uso de hipolipemiantes e que eles não faziam uso de outros medicamentos que potencialmente interferem no perfil lipídico. Nenhuma paciente do sexo feminino estava em uso de terapia de reposição hormonal (TRH).

O presente trabalho incluiu os seguintes grupos: grupo 1 (controle), constituído de 12 pacientes comprovadamente não-portadores de DAOP e DM2; grupo 2 (DAOP), constituído de 17 pacientes portadores de DAOP e não-portadores de DM2; grupo 3 (DM2), constituído de 18 pacientes portadores de DM2; grupo 4 (DM2 + DAOP), constituído de 19 pacientes portadores, simultaneamente, de DAOP e DM2. O tamanho mínimo da amostra ( $(n)$ foi definido utilizando-se o coeficiente de variação descrito na literatura para os diversos parâmetros avaliados neste estudo, considerando 5\% a 10\% de variação em torno da média e $2^{\circ}$ de liberdade $(g \mid)^{(3)}$. Para diagnóstico da DAOP foi utilizado o índice tornozelo-braquial (ITB), que foi calculado pela divisão do maior valor da pressão sistólica de uma das artérias do tornozelo pelo valor da pressão sistólica da artéria braquial. O resultado menor que 0,9 indicou a presença de $\operatorname{DAOP}^{(16,19)}$.

Foram excluídos da pesquisa pacientes com trauma vascular, trombose venosa profunda, insuficiência renal crônica, doença hepática, arteriopatias funcionais e arteriopatias não-ateroscleróticas confirmados por meio de exames clínicos e laboratoriais, como hemograma e dosagens de uréia, creatinina e bilirrubinas. Para a composição dos grupos controle e DAOP foram excluídos indivíduos com glicemia de jejum alterada ${ }^{(1)}$.

As amostras de sangue venoso foram obtidas depois de jejum de 12 horas, utilizando tubos do sistema Vacuttainer ${ }^{\circledR}$
(Becton \& Dickson) sem anticoagulante. As amostras foram centrifugadas a $2.500 \mathrm{rpm}$ durante 15 minutos e o soro foi aliquotado, identificado e estocado a $-70^{\circ} \mathrm{C}$ até o momento da realização dos testes.

O colesterol total e os triglicérides foram determinados pelos métodos enzimáticos colorimétricos Randox Cholesterol CHOD-PAP e Randox Triglycerides GPO-PAP, respectivamente. As determinações de HDL-C e colesterol da lipoproteína de baixa densidade (LDL-C) foram realizadas pelo método enzimático de eliminação da Randox HDL Cholesterol Direct e Randox LDL Cholesterol Direct, respectivamente. As dosagens de Apo A-I e Apo B foram realizadas pelo método de turbidimetria da Biotécnica Apolipoproteína A-I Turbidimetria e Biotécnica Apolipoproteína B Turbidimetria, respectivamente. Os níveis plasmáticos de $L p(a)$ foram obtidos pelo método de turbidimetria, utilizando o conjunto diagnóstico In Vitro Lipoproteína(a) Turbidimétrico. Os testes foram realizados no equipamento Cobas Mira Plus, utilizando-se soros controles comerciais para verificar o desempenho dos ensaios. Para a dosagem de $L p(a)$ foi utilizado o control liquid (Spin React). Para interpretação dos níveis de $L p(a)$ foram considerados elevados os valores acima de $30 \mathrm{mg} / \mathrm{dl}$, o qual está estabelecido na literatura e é utilizado como ponto de corte pelos clínicos.

$\mathrm{Na}$ análise estatística foi utilizado o teste de análise de variância (ANOVA) para aqueles resultados que apresentaram distribuição normal (CT, LDL-C, HDL-C e Apo A-I) e o teste de Kruskal-Wallis para os dados que apresentaram distribuição não-paramétrica [TG, Apo B e Lp(a)]. Os dados com distribuição normal são apresentados como média, enquanto os com distribuição não-paramétrica o são como mediana. Os programas Sigma Stat versão 1.0 e Prism ver- 
são 3.0 foram utilizados para realizar as análises estatísticas e construir os gráficos, respectivamente.

\section{Resultados}

Em relação aos fatores de risco para o desenvolvimento da DAOP, observa-se que o tabagismo e a hipertensão arterial sistêmica (HAS) foram mais prevalentes nesse grupo de pacientes, sendo que $88,2 \%$ daqueles com DAOP eram também tabagistas e/ou hipertensos. A presença de claudicação intermitente $(\mathrm{Cl})$ é um sintoma específico para DAOP e, portanto, não foi encontrada nos demais grupos, não-portadores dessa doença (controle e DM2). No grupo apenas com DAOP, esse sintoma foi relatado por $70,6 \%$ dos pacientes, enquanto apenas $36,8 \%$ com DM2 + DAOP relataram a presença de $\mathrm{Cl}$. A história prévia (HP) de acidente vascular ou cirurgia em membros inferiores foi mais prevalente no grupo com DAOP $(41,2 \%)$ em comparação com o grupo DM2 + DAOP (26,3\%). Após a análise desses fatores de risco foram encontradas diferenças estatísticas significativas entre os grupos controle e DAOP para os parâmetros hipertensão arterial sistêmica (HAS), tabagismo e HP, com valores de $p<0,001,<0,001$ e 0,023, respectivamente. Foi encontrada diferença estatística entre os grupos controle e
DM2 + DAOP para o fator de risco tabagismo, com valor de $p=0,032$. Para os demais parâmetros avaliados como fatores de risco (dislipidemia, história familiar, idade e sexo), não foram encontradas diferenças estatísticas significativas entre os grupos estudados (Tabela 1).

Os resultados obtidos para os parâmetros bioquímicos nos grupos estudados podem ser observados na Tabela 2. Não foram observadas diferenças estatísticas significativas entre as medianas dos níveis plasmáticos de $L p(a)$, porém valores mais elevados de $L p(a)$ foram encontrados nos pacientes com DAOP (grupos DAOP e DM2 + DAOP), inclusive com resultados acima do valor limítrofe $(30 \mathrm{mg} / \mathrm{dl})$. Esses grupos apresentaram valores de $L p(a)$ cerca de três e quatro vezes maiores que a mediana do grupo controle, respectivamente (Figura). Na avaliação do ITB, observouse uma relação inversa entre os níveis plasmáticos de $L p(a)$ e os valores do índice, ou seja, foi evidenciada correlação negativa entre $L p(a)$ e os valores do ITB (coeficiente de correlação $-0,3$ e valor de $p=0,015$ ).

A prevalência de níveis de $\mathrm{Lp}(\mathrm{a})$ maiores que $30 \mathrm{mg} / \mathrm{dl}$, obtida pelo teste exato de Fisher, foi de 42\%, 59\%, 39\% e $58 \%$, respectivamente, para os grupos controle, DAOP, DM2 e DM2 + DAOP. Não houve diferença significativa entre os grupos avaliados para prevalência de $\operatorname{Lp}(\mathrm{a})(p>0,05)$.

\section{Tabela 2 Parâmetros bioquímicos para os grupos estudados}

\begin{tabular}{|c|c|c|c|c|c|}
\hline Parâmetros & $\begin{array}{l}\text { Controle } \\
(n=12)\end{array}$ & $\begin{array}{l}\text { DAOP } \\
(n=17)\end{array}$ & $\begin{array}{l}\text { DM2 } \\
(n=18)\end{array}$ & $\begin{array}{l}\text { DM2 + DAOP } \\
(n=19)\end{array}$ & Valor de $p$ \\
\hline $\mathrm{CT}(\mathrm{mg} / \mathrm{dl})^{1}$ & $\begin{array}{l}193,83 \\
( \pm 34,4)\end{array}$ & $\begin{array}{l}200,33 \\
( \pm 49,82)\end{array}$ & $\begin{array}{l}196,39 \\
( \pm 72,49)\end{array}$ & $\begin{array}{l}178,89 \\
( \pm 40,09)\end{array}$ & ns \\
\hline HDL-C (mg/dl) $)^{1}$ & $\begin{array}{l}46,33^{a} \\
( \pm 10,51)\end{array}$ & $\begin{array}{l}39,73^{a, b} \\
( \pm 13,25)\end{array}$ & $\begin{array}{l}42,22^{\mathrm{a}} \\
( \pm 12,29)\end{array}$ & $\begin{array}{l}31,74^{\mathrm{b}} \\
( \pm 8,46)\end{array}$ & 0,004 \\
\hline LDL-C (mg/dl) $)^{1}$ & $\begin{array}{l}124,9 \\
( \pm 31,4)\end{array}$ & $\begin{array}{l}133,81 \\
( \pm 42,05)\end{array}$ & $\begin{array}{l}122,18 \\
( \pm 59,45)\end{array}$ & $\begin{array}{l}111,64 \\
( \pm 42,96)\end{array}$ & ns \\
\hline Apo A-I (mg/dl) $)^{1}$ & $\begin{array}{l}159,69^{a} \\
( \pm 10,3)\end{array}$ & $\begin{array}{l}152,35^{a, b} \\
( \pm 14,51)\end{array}$ & $\begin{array}{l}156,39 a, b \\
( \pm 9,57)\end{array}$ & $\begin{array}{l}146,29^{b} \\
( \pm 13,38)\end{array}$ & 0,019 \\
\hline $\mathrm{TG}(\mathrm{mg} / \mathrm{dl})^{2}$ & $\begin{array}{l}110,5 \\
(40-250)\end{array}$ & $\begin{array}{l}117 \\
(38-233)\end{array}$ & $\begin{array}{l}132,5 \\
(44-318)\end{array}$ & $\begin{array}{l}141 \\
(50-649)\end{array}$ & ns \\
\hline Apo B $(\mathrm{mg} / \mathrm{dl})^{2}$ & $\begin{array}{l}75,7 \\
(56,4-101,5)\end{array}$ & $\begin{array}{l}67,6 \\
(42,8-96)\end{array}$ & $\begin{array}{l}69,2 \\
(44,9-126,4)\end{array}$ & $\begin{array}{l}71,1 \\
(45-100,8)\end{array}$ & ns \\
\hline Lp(a) $(\mathrm{mg} / \mathrm{dl})^{2}$ & $\begin{array}{l}10,15 \\
(3,6-83,4)\end{array}$ & $\begin{array}{l}32,8 \\
(3,6-189,7)\end{array}$ & $\begin{array}{l}9,15 \\
(3,6-80,5)\end{array}$ & $\begin{array}{l}37,9 \\
(3,6-158,3)\end{array}$ & ns \\
\hline
\end{tabular}

${ }^{1}$ Resultados com distribuição normal expressos em média e desvio padrão; ${ }^{2}$ resultados com distribuição não-paramétrica expressos em mediana e mínimo e máximo; a bletras iguais entre si indicam ausência de diferença estatística, e letras diferentes, diferença estatística significativa entre as médias ou medianas.

DAOP: doença arterial obstrutiva periférica; DM2: diabetes mellitus tipo 2; $\mathrm{n}$ : número de indivíduos; CT: colesterol total; HDL-C: colesterol da lipoproteína de alta densidade; LDL-C: colesterol da lipoproteína de baixa densidade; Apo A-I: apolipoproteína A-l; TG: triglicérides; Apo B: apolipoproteína B; Lp(a): lipoproteína (a); ns: não-significativo. 


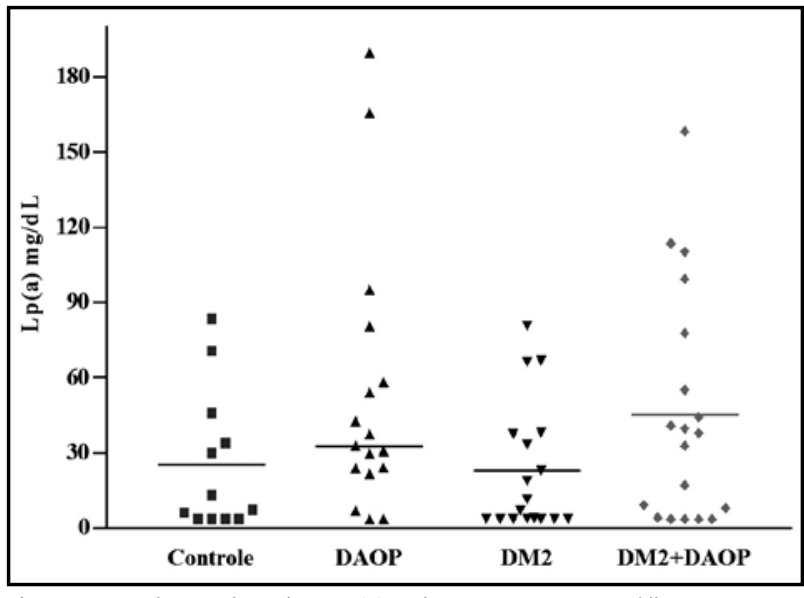

Figura - Distribuição dos valores $L p(a)$. Valores expressos em $\mathrm{mg} / \mathrm{dl}$ para os grupos controle, DAOP, DM2 e DM2 + DAOP. O valor desejável de $L p(a)$ é inferior a $30 \mathrm{mg} / \mathrm{dl}$. As linhas horizontais representam as medianas dos grupos. Não foi observada diferença significativa entre as medianas dos grupos estudados

Lp(a): lipoproteína (a); DAOP: doença arterial obstrutiva periférica; DM2: diabetes mellitus tipo 2.

Não foram encontradas diferenças significativas entre as médias dos níveis plasmáticos de CT e LDL-C e entre as medianas dos níveis plasmáticos de triglicérides. Os valores das medianas para TC e os valores das médias para CT, respectivamente, encontravam-se dentro das faixas de concentração desejáveis e/ou limítrofes ${ }^{(18)}$. Verificou-se elevação moderada de LDL-C nos grupos de pacientes diabéticos (DM2 e DM2 + DAOP), pois os valores desejáveis de LDL-C em indivíduos diabéticos são menores que $100 \mathrm{mg} / \mathrm{dl}^{(18)}$. Foi evidenciada correlação positiva significativa entre os resultados de LDL-C e Apo B, com coeficiente de correlação 0,64 e valor de $p<0,0001$, bem como foi observada correlação positiva entre $L p(a)$ e LDL-C, com coeficiente de correlação 0,43 e valor de $p=0,0004$. As médias obtidas para HDL-C apresentaram diferença significativa entre os grupos controle vs. DM2 + DAOP e entre os grupos DM2 vs. DM2 + DAOP. Observa-se que, nos grupos de pacientes diabéticos (DM2 e DM2 + DAOP), os valores das médias obtidas para HDL-C encontraram-se abaixo dos valores desejáveis, que são superiores a $45 \mathrm{mg} / \mathrm{dl}$ para diabéticos ${ }^{(18)}$, com redução significativa no grupo DM2 + DAOP. Os resultados obtidos para HDL-C e triglicérides apresentaram correlação negativa significativa com coeficiente de correlação -0,31 e valor de $p=0,0131$. Foi observada diferença estatística significativa para a relação $\mathrm{CT} / \mathrm{HDL}-\mathrm{C}$ entre os grupos controle vs. DM2 + DAOP, $\operatorname{com} p=0,023$.

As médias obtidas para Apo A-I apresentaram diferença significativa entre os grupos controle vs. DM2 + DAOP, entretanto os valores de todas as médias de Apo A-I estão na faixa de referência do método. Foi observada correla- ção positiva entre os resultados de HDL-C e Apo A-I, com coeficiente de correlação 0,819 e valor de $p<0,0001$. Na avaliação de Apo $B$ não foram encontradas diferenças significativas entre as medianas dos grupos estudados. Todos os valores também se encontraram na faixa de referência do método. Foi observada correlação positiva entre os níveis de apo B e triglicérides com coeficiente de correlação $r=$ 0,53 e valor de $p<0,0001$.

\section{Discussão}

Vários estudos científicos evidenciam a relação entre alterações do perfil lipídico e desenvolvimento da aterosclerose. Os níveis plasmáticos de colesterol e triglicérides podem ser utilizados para identificação dos pacientes com elevado risco para desenvolver evento cardiovascular ${ }^{(15)}$. No entanto esses parâmetros falharam na identificação prévia de indivíduos que desenvolveram IAM, segundo pesquisas realizadas com pacientes norte-americanos ${ }^{(17)}$. Esses pacientes apresentaram níveis plasmáticos de colesterol e triglicérides normais ou apenas moderadamente elevados. O reconhecimento de que a aterosclerose é uma doença inflamatória de origem multifatorial, e não simplesmente resultado de acúmulo lipídico nos vasos sangüíneos, permitiu a descoberta e a validação de novos parâmetros bioquímicos para a predição e o acompanhamento da DAOP e de outras doenças vasculares, principalmente naqueles pacientes normolipêmicos.

Neste estudo não foram encontradas diferenças estatísticas significativas entre as médias dos níveis plasmáticos de colesterol total, LDL-C e entre as medianas dos níveis plasmáticos de triglicérides (Tabela 2). Os valores das respectivas médias e medianas para esses parâmetros encontram-se nas faixas desejáveis e limítrofes ${ }^{(18)}$. Em relação à fração LDL-C é importante considerar que a maior preocupação em relação a essa lipoproteína, no diabético, é que as diferenças parecem ser mais qualitativas que quantitativas. Verifica-se na literatura a indicação de alta prevalência de partículas pequenas e densas em indivíduos diabéticos, mesmo quando normolipêmicos, as quais estão associadas ao aumento de risco cardiovascular ${ }^{(13)}$.

As médias obtidas para HDL-C apresentaram diferença significativa entre os grupos controle vs. DM2 + DAOP e entre DM2 vs. DM2 + DAOP. Observa-se na Tabela 2 que, nos grupos DM2 e DM2 + DAOP, os valores das médias obtidas para HDL-C encontraram-se abaixo do desejável(18), com redução significativa no grupo DM2 + DAOP, demonstrando a existência de um menor benefício antiaterogênico 
nesses grupos devido à redução da fração HDL-C, fato já descrito na literatura ${ }^{(13)} \mathrm{e}$, portanto, maior associação com risco cardiovascular. Acredita-se que a redução dos níveis de HDL-C nos pacientes diabéticos deve-se a um menor fornecimento de elementos que formam essa lipoproteína originada do metabolismo de quilomícrons e lipoproteína de muito baixa densidade (VLDL), entretanto um possível mecanismo para essa redução ainda não está bem esclarecido ${ }^{(13)}$.

A relação $\mathrm{CT} / \mathrm{HDL}-\mathrm{C}$ foi capaz de distinguir o grupo DM2 + DAOP, que apresentou diferença estatística significativa $(p=0,023)$ em relação ao grupo controle. Essa relação é considerada um bom marcador para o desenvolvimento de doença vascular ${ }^{(16)}$ e pode estar associada a um risco aumentado de aparecimento e agravamento da DAOP.

Não foram encontradas diferenças significativas entre as medianas das concentrações plasmáticas de Apo B. Entretanto, as médias obtidas para Apo A-I apresentaram diferenças significativas entre os grupos controle e DM2 + DAOP. Todos os valores das médias e medianas obtidas para Apo B e Apo A-I encontraram-se na faixa de referência preconizada ${ }^{(18)}$. As correlações positivas observadas entre HDL-C e apo A-I e entre LDL-C e Apo B já eram esperadas, uma vez que a principal apolipoproteína constituinte do HDL-C é a Apo A-I, e do LDL-C, a Apo B. Entretanto, estabelecer a magnitude da correlação torna-se importante para validação dos testes que não são empregados rotineiramente pelos laboratórios clínicos, como é o caso das dosagens de Apo A-I e Apo B. Os níveis de apolipoproteína têm sido descritos como melhores preditores de doença coronariana que as lipoproteínas( ${ }^{(8)}$. As concentrações plasmáticas de apolipoproteínas são determinadas geneticamente e sofrem pouca influência de variáveis biológicas. Já os lípides apresentam flutuação de níveis plasmáticos em torno dos seus pontos de equilíbrio em resposta aos vários estímulos do controle metabólico ${ }^{(12)}$. Os resultados obtidos neste estudo demonstraram que as frações do colesterol, LDL-C e HDL-C, destacaram melhor as tendências para risco aumentado de aterosclerose e evento vascular agudo quando em comparação com as apolipoproteínas, Apo B e Apo A-I, respectivamente, especialmente nos grupos portadores de DAOP.

Com relação aos valores de $L p(a)$, observa-se que as medianas obtidas para os grupos DAOP e DM2 + DAOP encontraram-se visivelmente acima dos valores de referência $(<30 \mathrm{mg} / \mathrm{dl})$, apresentando medianas cerca de três a quatro vezes maiores que a do grupo controle, respectivamente. Porém, não foram observadas diferenças significativas entre as medianas dos grupos estudados. Neste estudo, a resposta obtida para a $L p(a)$ foi muito variada, pois foram encontrados níveis plasmáticos muito baixos (mínimos) e muito elevados (máximos) dentro de um mesmo grupo. Esse resultado mostra que a $L p(a)$ é um parâmetro extremamente variável, que depende da resposta individual de cada paciente, e isso pode ter influenciado a ausência de diferenças significativas entre as medianas dos grupos estudados. No entanto, como houve tendência a maior elevação de $L p(a)$, inclusive acima dos níveis de referência, no grupo com DAOP e DM2 + DAOP, esse parâmetro pode ser considerado um marcador com potencial valor para sinalizar maior risco de aterosclerose e evento cardiovascular nesses pacientes.

Embora não tenham sido observadas diferenças significativas quanto à prevalência de níveis elevados de $L p(a)$ entre os quatro grupos estudados, a elevação dessa lipoproteína nos grupos DAOP e DM2 + DAOP demonstra maior percentual de prevalência (59\% e $58 \%$, respectivamente).

A relação inversa entre a redução do ITB (à medida que esse decresce, a manifestação clínica da DAOP pode ser agravada) e o aumento da $L p(a)$ reforça a idéia de que esse último parâmetro pode ser uma ferramenta adicional prognóstica importante.

A maior prevalência do sintoma claudicação intermitente $(\mathrm{Cl})$ no grupo DAOP, em comparação com o grupo DM2 + DAOP, pode ser justificada pelo fato de que o grupo apenas com DAOP apresentou, após análise global de todos os parâmetros, alterações laboratoriais mais pronunciadas e maior porcentagem de fatores de risco, em comparação com o grupo com DM2 + DAOP.

Quanto às limitações do estudo, apesar de o tamanho da amostra ser estatisticamente correto ${ }^{(3)}$, deve-se considerar que o $n$ amostral não representa o universo de pacientes diabéticos, uma vez que se têm milhões de pessoas com DM2 em todo o mundo. Além disso, não foi possível parear os grupos estudados em relação ao índice de massa corporal (IMC), importante fator a ser considerado devido à associação existente entre obesidade e dislipidemia. Por fim, o tempo médio de diagnóstico para os pacientes diabéticos foi de 11,1 anos, porém a faixa encontrada para o tempo de diagnóstico foi de 2 a 34 anos, e essa diferença também poderia interferir nos resultados.

Acredita-se que um maior conhecimento sobre os fatores que agravam a DAOP possibilitará a adoção de medidas profiláticas, visando reduzir a ocorrência de diversas manifestações oclusivas vasculares e eventos trombóticos agudos, especialmente os cardiovasculares, coronarianos e cerebrovasculares. 


\section{Conclusão}

Para as variáveis lipídicas estudadas foram observadas diferenças estatísticas significativas apenas entre os níveis plasmáticos de HDL-C e Apo A-I.

Para o parâmetro $L p(a)$ foram observados níveis plasmáticos mais elevados nos grupos DAOP e DM2 + DAOP, respectivamente, os quais também apresentam uma concomitante e significativa redução de HDL-C.

O aumento dos níveis plasmáticos de $L p(a)$ sugere que essa lipoproteína pode estar associada à presença da
DAOP e que poderia ter um papel relevante e adicional na prevenção da mesma.

Essa linha de pesquisa representa um desafio para várias áreas de estudo de avaliação do envolvimento da Lp(a) em diversos tipos de eventos ateroscleróticos, pois os mecanismos de aumento de risco não são completamente conhecidos. Outros estudos são necessários para obter melhor compreensão sobre a participação desse marcador e de outros fatores na patogênese e na progressão da aterosclerose.

\section{Referências}

1. ADA. Follow-up report on the diagnosis of diabetes mellitus: American Diabetes Association. Diabetes Care, v. 26, 2003.

2. ANGLÉS-CANO, E.; PEÑA-DÍAZ, A.; LOYAU, S. Inhibition of fibrinolysis by lipoprotein(a). Ann N Y Acad Sci, v. 936, p. 261-75, 2001.

3. ARMITAGE, P. Statistical methods in medical research. London: Blackwell Scientific Publishers, 1973.

4. BERG, K. A new serum type system in man: the Lp system. Acta Pathol Scand, v. 59, p. 382-6, 1963.

5. DURIEZ, P.; DALLONGEVILLE, J; FRUCHART, J. C. Lipoprotein(a) as a marker for coronary heart disease. Br J Clin Pract Suppl, v. 77, n. 1, supl. A, p. S54-61, 1996.

6. HAJJAR, K. A.; NACHMAN, R. L. The role of lipoprotein(a) in atherogenesis and thrombosis. Ann Rev Med, v. 47, p. 423-42, 1996.

7. HANCOCK, M. A. et al. Inhibition of plasminogen activation by lipoprotein(a): critical domains in apolipoprotein(a) and mechanism of inhibition on fibrin and degraded fibrin surfaces. J Biol Chem, v. 278, p. 23260-9, 2003.

8. HSIA, S. L. et al. Serum levels of high-density lipoprotein phospholipids correlate inversely with severity of angiographically defined coronary artery disease. Atherosclerosis, v. 152, p. 469-73, 2000.

9. JUNQUEIRA, V. B. C.; GOMES, L. F. Homocysteine and vitamins: risk factors for cardiovascular disease. Disponível em: <http://www.helthandage.com/html/ res/controversies/content/chap4/homocysteine_ful3_ es.htm>. Acesso em: 30 jan. 2003.

10. KOSCHINSKY, M. L. Lipoprotein(a) and the link between atherosclerosis and thrombosis. Can J Cardiol, v. 20, n. 8, supl. B, p. 37B-43B, 2004.
11. MARTINEZ, T. L. R. Condutas clínicas nas dislipidemias. Belo Horizonte: Heath, 1997. 291p.

12. MARTINEZ, T. L. R. Dislipidemias: da teoria à prática. São Paulo: Atheneu, 2004.

13. MARTINEZ, T. L. R. Manual de condutas clínicas em dislipidemias. Rio de Janeiro: Medline, 2003.

14. MILIONIS, H. J.; WINDER, A. F.; MIKHAILIDIS, D. P. Lipoprotein(a) and stroke. J Clin Pathol, v. 53, p. 48796, 2000.

15. RIFAI, N.; RIDKER P. M. High-sensitivity C-reactive protein: a novel and promising marker of coronary heart disease. Clin Chem, v. 47, p. 403-11, 2001.

16. SCHMIEDER, F. A.; CAMEROTA, A. J. Claudicação intermitente: magnitude do problema, avaliação do paciente e estratégias terapêuticas. Am J Cardiol, v. 87, p. 3-14, 2001.

17. SNIDERMAN, A. D. et al. Apolipoproteins versus lipids and indices of coronary risk and as targets for statin treatment. Lancet, v. 361, n. 1, p. 777-80, 2003.

18. SOCIEDADE BRASILEIRA DE CARDIOLOGIA. III Diretrizes Brasileiras Sobre Dislipidemias e Diretrizes de Prevenção da Aterosclerose. Arq Bras Cardiol, v. 77, supl. III, p. 1-48, 2001.

19. TASC (TransAtlantic Inter-Society Consensus) Working Group. Management of peripheral arterial disease (PAD). J Vasc Surg, v. 31, supl. 5-44, p. 54-74; 77122, 2000.

20. WHO (WORLD HEALTH ORGANIZATION). Health topics. Disponível em: <http://www.who.int/cardiovascular diseases/en/> 2004. Acesso em: 06 nov. 2004.

21. YAMADA, T. et al. Importance of hypercoagulability over hyperglycemia for vascular complication in type 2 diabetes. Diabetes Res Clin Pratice, v. 49, n. 1, p. 23-31, 2000. 